\title{
L'organisation de la lutte anti-terroriste aux Etats-
} Unis

\section{Philippe Bonditti}

\section{(2) OpenEdition}

1 Journals

Édition électronique

URL : http://journals.openedition.org/conflits/742

DOI : $10.4000 /$ conflits.742

ISSN : $1777-5345$

Éditeur :

CCLS - Centre d'études sur les conflits lilberté et sécurité, L'Harmattan

Édition imprimée

Date de publication : 1 décembre 2001

Pagination : 65-76

ISBN : 2-7475-2227-X

ISSN : 1157-996X

Référence électronique

Philippe Bonditti, «L'organisation de la lutte anti-terroriste aux Etats-Unis », Cultures \& Conflits [En ligne], 44 | hiver 2001, mis en ligne le 04 février 2003, consulté le 30 mars 2021. URL : http://

journals.openedition.org/conflits/742 ; DOI : https://doi.org/10.4000/conflits.742

Ce document a été généré automatiquement le 30 mars 2021.

Creative Commons License 


\title{
L'organisation de la lutte anti- terroriste aux Etats-Unis
}

\author{
Philippe Bonditti
}

De nos jours, la vision dominante du «terrorisme » le considère comme un phénomène objectivable, qui existe en soi, et dont les attentats et autres prises d'otages seraient l'expression en même temps que la preuve de son existence « réelle ». Elle le pose alors comme une menace à la sécurité des individus et à la sécurité nationale d'un pays. Face à elle, la réponse sécuritaire se fixe pour objectif d'en identifier les auteurs pour les anéantir.

En pointant le problème de la violence dite terroriste, acteurs politiques et institutionnels proposent des solutions qui passent par la nécessaire qualification des actes. Le terme terrorisme est ainsi utilisé comme un label pour qualifier les pratiques de violences politiques de certains individus ou groupes dans un jeu de rapport de force et d'opposition. La signification couramment attribuée à ce terme - qui ne renvoie à aucune réalité définie et unifiée - travestit en fait son sens historique en inversant la perspective. En effet, historiquement, le terrorisme renvoie aux pratiques étatiques d'instauration d'un état de terreur. Il est aujourd'hui utilisé pour qualifier les pratiques de violences dirigées contre les Etats ${ }^{1}$. L'emploi de ce terme à des fins de labellisation doit nous inciter à considérer le «terrorisme " comme une construction sociale dans laquelle les pratiques discursives des acteurs de l'anti-terrorisme notamment, tiennent une place centrale. Ces derniers ne sont pas concernésde facto par la problématique terroriste. Ils se présentent comme tels, en proposant leur vision du phénomène, en prétendant posséder le savoir utile qui va justifier leur implication dans la réponse qu'ils estiment devoir apporter au problème de la violence politique.

Il convient donc de recouper les pratiques discursives des acteurs avec la logique, les objectifs et les implications, notamment budgétaires, de leurs missions respectives. Ceux qui s'estiment concernés par la problématique terroriste, se placent de fait dans une situation de compétition pour l'attribution de budgets et, en amont, pour faire valoir leur approche du terrorisme. Et c'est à celui qui parvient à la rendre légitime aux yeux de l'opinion publique et des décideurs politiques, que sont attribués non 
seulement les budgets mais une place prépondérante dans l'architecture de l'antiterrorisme.

Bien qu'il ne concentre pas à lui seul, loin de là, l'ensemble des activités antiterroristes, nous centrerons ici notre attention sur le champ des professionnels de la sécurité aux Etats-Unis² ${ }^{2}$. Mandatés pour sécuriser les individus ou le territoire national, les acteurs de la sécurité tirent la légitimité de leurs actions et de leur existence même, de l'existence d'un ennemi et de la menace qu'il fait peser. Ils ont donc d'excellentes raisons d'exagérer les périls. Cela tient d'ailleurs plus à des raisons structurelles liées à leur rôle dans le système qu'à une volonté de tromper. La logique sécuritaire est extrême. Pour peu que les acteurs y participent avec un intérêt sincère, ils trouveront toujours des menaces contre lesquelles ils jugeront nécessaire de protéger ceux dont ils ont en charge la sécurité.

Avec les années 80, le label terroriste est, aux Etats-Unis, devenu un moyen de justifier l'attribution des budgets. Il s'est de ce fait instauré une véritable lutte pour l'usage légitime du label terroriste observable à travers la diversité des définitions utilisées du terrorisme.

\section{L'absence d'approche commune et le statut deLead Agency}

Depuis les années 1970, un réseau complexe de programmes et d'activités a été développé aux Etats-Unis pour dire et combattre le "terrorisme». Plus d'une quarantaine de départements, d'agences, de services et de bureaux participent aujourd'hui à la réponse qui doit lui être apportée. L'objectif de lutte que s'est fixé l'administration américaine se heurte, depuis ces années là, à l'absence de définition commune du terrorisme.

Le département d'Etat (DOS) utilise la définition donnée dans le titre 22 du code des Etats-Unis : "violence préméditée, à motivations politiques, exercée contre des cibles noncombattantes par des groupes sub-nationaux ou des agents clandestins, dont le but est généralement d'influencer une opinion $»^{3}$. Cette définition insiste sur le caractère prémédité, planifié et fondamentalement politique du terrorisme ainsi que sur sa nature « sub-nationale ». Le Federal Bureau of Investigation (FBI) se réfère quant à lui au Code of Federal Regulations et définit le terrorisme comme "l'usage illégal, ou la menace de faire usage de la force ou de la violence, par un groupe ou un individu basé et opérant entièrement à l'intérieur des Etats-Unis et de ses territoires sans ordre venu de l'étranger, contre des personnes ou des biens, pour intimider ou contraindre un gouvernement, la population civile, ou une partie de celle-ci, dans le but de promouvoir des objectifs politiques ou sociaux $»^{4}$. Cette définition est plus large que celle utilisée par le DOS. Elle pointe les objectifs sociaux tout autant que politiques du terrorisme et identifie des catégories-cibles plus larges que les seuls non-combattants : les gouvernements, les citoyens mais aussi les biens publics et privés. Pour le département de la Défense (DOD) le terrorisme est «l'usage illégal - ou la menace - de la force ou de la violence contre des individus ou des biens, pour contraindre ou intimider des gouvernements ou des sociétés, souvent pour atteindre des objectifs politiques, religieux ou idéologiques $»^{5}$. La particularité de cette définition tient au fait qu'elle insiste tout à la fois sur les buts religieux, idéologiques et politiques du terrorisme. Si la CIA semble aujourd'hui utiliser la même définition du terrorisme que le DOS $^{6}$, elle a néanmoins longtemps fait usage de sa propre définition : "la menace ou l'usage de la violence à des fins politiques par des individus ou groupes, s'ils agissent pour, ou en opposition à, un gouvernement établi, lorsque ces actions ont pour dessein d'influencer un groupe-cible plus large que la ou les seules victimes $»^{7}$. Enfin, le FBI, le DOS et la CIA, dont les 
activités dépassent le cadre des frontières nationales du pays, possèdent chacun une définition internationale du terrorisme.

Toutes ces définitions traduisent en fait les priorités et les intérêts spécifiques des acteurs concernés et leur permettent de justifier leur implication dans la lutte contre le terrorisme. La distinction établi entre « terrorisme » et « terrorisme international » est un premier révélateur de l'instrumentalisation de la violence politique. Elle permet en effet au département d'Etat, un acteur d'abord tourné vers l'extérieur et de surcroît responsable de la sécurité de ses diplomates, de s'impliquer dans l'anti-terrorisme. Ces définitions sont par ailleurs révélatrices d'une tension entre le travail d'investigation et d'application de la loi du FBI, et celui plus politique et diplomatique du département d'Etat. Le FBI a ainsi intérêt à ouvrir un champ aussi vaste que possible qui relève de sa responsabilité. Il insiste donc sur l'acte de violence effectif en même temps que sur la menace. La seule essence politique de cette violence ne suffit par ailleurs pas. Elle est donc totalement diluée dans une approche plus large qui pointe les motivations sociales d'exercice d'une violence politique. A l'inverse, le département d'Etat opte pour une définition vague et très politique, qui lui permet de disposer d'une certaine marge de manœuvre et d'utiliser le label terroriste en fonction des contraintes diplomatiques qui se posent à lui. De la même manière, le DOD a intérêt, pour donner une consistance à sa responsabilité et assurer pleinement la sécurité de ses hommes à l'étranger,à pouvoir qualifier de terroriste un éventail assez large d'actes de violence. Il insiste donc, lui aussi, sur l'acte en lui-même et la menace, et associe au terrorisme des raisons religieuses et idéologiques.

L'implication progressive d'un nombre toujours plus important d'acteurs, aux approches différentes, dans la lutte contre le terrorisme explique qu'il n'y ait jamais eu, aux Etats-Unis, de politique anti-terroriste cohérente mais une superposition d'approches. Dès 1985, s'est ainsi posé la question de leur coopération et de la coordination de leurs efforts. Le statut de Lead Agency, autour duquel se structure l'architecture de l'anti-terrorisme depuis le début des années 1980, a alors revêtu une acuité particulière.

\section{Le statut de Lead Agency}

Ce statut confère à l'agence qui en est bénéficiaire des responsabilités élargies en matière de coordination, de procédure et de programmation de la réponse antiterroriste. Selon les termes de la doctrine militaire, «l'agence-leader» est désignée parmi les agences gouvernementales américaines pour coordonner l'ensemble des activités des différentes agences dans la conduite quotidienne d'une opération en cours. Elle dirige le groupe de travail inter-gouvernemental mis en place pour coordonner la politique relative à la dite opération, détermine l'agenda, assure la cohésion entre les agences et est responsable de l'exécution des décisions. Présenté comme le moyen d'assurer la coordination des divers acteurs engagés dans l'antiterrorisme et rendre ainsi plus efficace la réponse qui doit lui être apportée, un tel statut hiérarchise le champ de l'anti-terrorisme. Et c'est bien là que réside l'enjeu réel de l'attribution de ce statut. Octroyer de telles responsabilités au FBI ou au Pentagone n'a pas les mêmes implications, et la désignation de telle ou telle agence Lead Agency constitue donc un choix, déjà décisif, dans l'orientation de toute politique antiterroriste. En se voyant investi du pouvoir d'approuver ou de rejeter les propositions d'autres agences, l'agence-leader occupe de ce fait une position prépondérante depuis laquelle elle peut influer sur la mise en pratique de la politique anti-terroriste 
déterminée au sein du NSC. Loin de résoudre les problèmes de coopération, ce statut pose le risque de faire primer une réponse qui n'intègre que les préoccupations des agences titulaires d'un tel statut au détriment de l'efficacité, pourtant recherchée, du traitement global de la problématique terroriste ${ }^{8}$.

Mais ce statut ne prend véritablement sens qu'au regard de la complexe architecture de l'anti-terrorisme aux Etats-Unis et de son évolution depuis la fin des années 1960. La politique américaine de lutte anti-terroriste a pour la première fois été formalisée par la directive de sécurité nationale 207, signée par le président Reagan en 1986. Estimant que leur pays était, à l'époque, d'abord victime du terrorisme à l'étranger, responsables politiques et bureaucratiques ont alors fait le choix d'axer la lutte contre le terrorisme dit « international » et le DOS a ainsi été réaffirmé dans ses prérogatives de Lead Agency.

\section{La répartition des compétences}

Le rôle du DOS

C'est en 1972 que le président Nixon a pour la première fois impliqué le DOS dans la lutte contre le terrorisme en nommant un assistant spécial auprès du Secrétaire d'Etat (Special Assistant to the Secretary of State and Coordinator of the Office for Combating Terrorism). En tant que responsable de l'office for Combating Terrorism(OCT), il dirige et coordonne, depuis, les travaux de l'Interagency Working Level Committeechargé, au niveau fédéral, d'apporter une réponse au terrorisme. En 1982, signe d'une implication plus forte du DOS dans l'anti-terrorisme, l'OCTa récupéré la réalisation de l'unique rapport annuel sur le terrorisme international jusqu'alors rédigé par la CIA. La directive 207 de 1986 n'a donc fait qu'entériner la place du DOS dans l'anti-terrorisme.

L'implication du département d'Etat tient, pour une part, à cette distinction effectuée entre « terrorisme indigène » et « terrorisme international » et à l'instrumentalisation de cette approche qui lui confère une position tout à fait singulière. Mais elle tient aussi à des éléments d'ordre événementiel. Ainsi, la prise d'otage à l'ambassade des EtatsUnis en Iran en 1979. Elle a particulièrement marqué les diplomates américains qui n'ont eu de cesse, jusqu'au début des années 1990, d'évoquer cet événement pour justifier la reconduction des budgets, notamment consacrés à la protection du personnel expatrié. Les attentats contre les ambassades américaines, en août 1998, au Kenya et en Tanzanie, ont également renforcé l'idée d'un DOS placé en première ligne face au terrorisme. Les responsables du département d'Etat ont longtemps joué de cette position de leur administration pour conserver la place qui est la leur dans l'organigramme des acteurs impliqués dans la lutte anti-terroriste.

Le DOS est ainsi, jusqu'au milieu des années 1990, le principal département à dessiner les lignes d'une politique anti-terroriste. Grâce à son Office for Combating Terrorism, renommé en 1985 Office for Counterterrorism, ce département s'est assuré une sorte de leadership dans l'anti-terrorisme.

Si la distinction terrorisme indigène/terrorisme international ne présente aucune pertinence pour l'analyste, il n'en demeure pas moins qu'elle est, aux Etats-Unis, un élément structurant dans l'élaboration d'une politique de lutte anti-terroriste. Les attentats perpétrés contre le World Trade Center en 1993 et le bâtiment fédéral d'Oklahoma City en 1995, ont amené l'administration américaine à resserrer l'activité anti-terroriste autour de la menace intérieure. Si les directives présidentielles 39 et 62, signées par le président Clinton en 1995 et 1998, ont réaffirmé le statut de Lead Agency du département d'Etat, elles ont aussi donné une deuxième tête pensante à la politique anti-terroriste: le FBI. Ce dernier va marquer l'anti-terrorisme de son empreinte et 
orienter, dès 1995, les efforts de lutte contre la «cyber-menace ». La directive 39 a par ailleurs posé trois éléments de stratégie nationale contre le terrorisme :

- réduire la vulnérabilité des Etats-Unis face aux attaques terroristes pour prévenir et empêcher les attentats ;

- répondre à ces attaques, appréhender et punir leurs auteurs ;

- gérer les conséquences des attentats.

Cette même directive a aussi défini le rôle de chacun des acteurs jugé utile à la réponse anti-terroriste. Chaque département a ainsi en charge la sécurité de tout ce qui relève de sa juridiction. Le département des transports assure la sécurité de tous les moyens et infrastructures de transport, aux Etats-Unis et à l'étranger; le DOS, celle des personnels, équipements et infrastructures non militaires américains à l'étranger; le DOD, celle du personnel, des infrastructures militaires et des équipements américains, aux Etats-Unis comme à l'étranger. Le département du Trésor (DOT) est chargé de la sûreté des flux et échanges financiers et veille à ce que les fonds en circulation n'aient pas pour objectif le financement d'organisations terroristes. Pour cela, il travaille en collaboration avec les douanes et administre les sanctions contre les Etats dits sponsors du terrorisme. C'est enfin au DOT d'assurer, par l'intermédiaire des services secrets qui relèvent de sa juridiction - la protection du Président et des hauts responsables de l'administration américaine aux Etats-Unis et à l'étranger. Le FBI et le DOS doivent alors composer avec cet acteur.

En cas d'attaque terroriste, le NSC est, dans l'urgence, l'organe central de coordination de tous ces acteurs et c'est à lui que revient la responsabilité d'activer les statuts de Lead Agency du département d'Etat ou du FBI, pour la mise en œuvre des opérations.

Le renseignement : $\mathrm{FBI}$, $\mathrm{CIA}$

Le renseignement a toujours été présenté comme un élément indispensable et le premier outil de la lutte anti-terroriste. Particulièrement à l'œuvre au premier niveau de stratégie, les logiques de renseignement doivent permettre l'accumulation d'un savoir sur l'ennemi terroriste pour anticiper les actes de violence. L'Interagency Intelligence Committee on Terrorism rassemble, à cette fin, plus de quarante départements, agences, services et bureaux. Cette structure doit permettre une mise en partage des informations recueillies par ces différents acteurs. Parmi eux, deux sont prépondérants en matière de renseignement pour tout ce qui relève du terrorisme : le FBI, au niveau national, et la CIA, au niveau international.

L'implication du FBI dans la lutte anti-terroriste date du début des années 1980. William H. Webster, alors directeur du Bureau, décide, en 1982, de faire du contreterrorisme la quatrième priorité nationale du FBI. Dès 1984, cette agence est nommée Lead Agency pour la sécurité des Jeux olympiques de Los Angeles. Puis elle est autorisée, par le Congrès, en 1986, à enquêter sur des attentats terroristes commis contre des ressortissants américains à l'étranger. Entre 1990 et 1996, 500 agents supplémentaires sont venus gonfler ses services de renseignement et de lutte contre le terrorisme. Ces deux activités ont d'ailleurs été placées au centre de son programme de sécurité nationale en 1992. Ses activités de renseignement s'orientent depuis 1996 vers les menaces terroristes d'origine nationale, étrangère et virtuelle («cyber-menace »). Le 
Computer Investigation and Infrastructure Threat Assessment Center a, cette même année, été inauguré pour répondre aux menaces physiques et virtuelles dirigées contre des infrastructures américaines. Il a été remplacé en 1998 par le National Infrastructure Protection Center, une structure plus large (56 postes supplémentaires) et au budget plus important de 6 millions de dollars. Entre 1995 et 1998, à la faveur de la loi antiterroriste votée en 1996 et qui porta le budget de la lutte contre le terrorisme à 11 milliards de dollars, le «budget anti-terroriste» du FBI a ainsi plus que doublé et s'élève aujourd'hui à 580 millions de dollars 9 .

Responsable des activités de renseignement à l'extérieur des Etats-Unis, la CIA a été le premier organe à produire un rapport annuel sur «l'évolution du terrorisme international » entre 1968 et 1982. Elle dispose, depuis 1986, du Counterterrorism Center où est notamment représentée la National Security Agencypar ailleurs responsable du programme Echelon - et qui collecte, analyse et distribue aux acteurs concernés toutes les informations susceptibles de servir pour pénétrer et détruire les organisations terroristes. Depuis 1992, la CIA porte, comme le FBI, un intérêt tout particulier aux nouvelles technologies et aux « risques » de voir les terroristes en faire usage. A côté de ses activités de renseignement, elle mène également des opérations secrètes visant à influer sur des événements dans des pays étrangers. Si le Congrès a attendu 1996 pour légaliser l'emploi de telles méthodes, en exhortant même le président à y recourir, ces pratiques étaient devenues un véritable instrument de politique étrangère sous l'administration Reagan. Le budget total prévu pour ces opérations s'est élevé à 2 milliards de dollars en 1986. Il s'élève depuis à 1 milliard de dollars chaque année sans compter les coûts de fonctionnement ${ }^{10}$. Mais le chiffre global consacré aux opérations secrètes est bien plus élevé encore car le Pentagone est aussi responsable d'un certain nombre de ces actions.

\section{L'emploi de la force armée}

L'acteur militaire s'est en effet progressivement impliqué dans la lutte anti-terroriste à partir du début des années 1980. Arguant alors de l'inadaptation des forces armées américaines face aux mouvements insurrectionnels pro-communistes du Tiers Monde, les hauts responsables militaires du Pentagone ont développé, à partir de la théorie de la contre-insurrection des années 1960, une doctrine élargie dite des « conflits de basse intensité ${ }^{11}$ ». Mais cette doctrine, qui regroupait sous cette même appellation de LIC des formes pourtant variées de conflictualité (prises d'otages, attentats terroristes, guérillas, lutte contre le trafic de drogue, opérations de maintien de la paix...), ne proposait pas seulement une nouvelle grille de lecture des conflits. Elle définissait aussi un spectre très large de missions dans lequel elle a intégrée la lutte contre le terrorisme, et prévoyait le développement d'une nouvelle catégorie de force : les Special Operation Forces. Préparées et équipées pour faire face aux LIC et à l'expansionnisme soviétique, certaines d'entre-elles ont été spécialement créées pour répondre aux attaques dites terroristes.

Le discours des hauts responsables militaires de l'époque entrait en parfaite résonance avec la volonté de l'administration Reagan de rompre avec la doctrine du Containment et d'adopter une posture offensive, celle du roll-back. Faisant valoir sa capacité à frapper, le Pentagone s'est imposé comme le bras armé de toute politique de lutte antiterroriste et s'est ainsi invité au partage du gâteau budgétaire confectionné dans cet objectif. Avec la disparition de l'ennemi soviétique, la doctrine LIC s'est fondue dans la doctrine Lake développée dès $1992^{12}$. Cette dernière a plus clairement encore associé la 
menace terroriste à quelques Etats et notamment les Rogues States, accusés de soutenir le terrorisme international.

Le recours à l'acteur militaire est essentiellement prévu au premier niveau de stratégie, via les opérations secrètes, pour détruire des bases terroristes à l'étranger, et au second, dans le cas d'attaques terroristes à l'étranger, par des accords de coopération avec le département d'Etat pour l'évacuation des citoyens américains. En cas de " grave menace» ou d'«attentat exceptionnel» qui dépasse les capacités du FBI, l'acteur militaire peut aussi intervenir sur le sol américain, en exception au Posse Comitatus Act. L'Attorney General doit alors formuler une demande explicite d'aide auprès du Secrétaire de la Défense. Un transfert de contrôle des opérations s'effectue alors du FBI vers le commandement militaire le temps de son intervention. Il existe enfin deux précédents à l'implication de l'acteur militaire en représailles à des attaques dites terroristes : en 1986, contre la Libye, suite à un attentat contre une discothèque à Berlin, et en 1993, contre l'Irak, après que le services secrets américains aient soi-disant acquis la preuve que Saddam Hussein avait commandité l'assassinat de Georges Bush père lors d'une visite officielle au Koweït.

De tout cela, nous pouvons tirer plusieurs enseignements :

D'abord le nombre très important d'acteurs impliqués dans l'anti-terrorisme et les difficultés à coordonner leurs activités. L'inefficacité dénoncée des services de renseignement depuis les événements du 11 septembre, tient pour une part à cette impossible coordination. Depuis vingt ans, les diverses productions législatives ou exécutives ont en fait créé des "mécanismes bureaucratiques de gestion» de la problématique terroriste peu adaptés aux réalités du problème. La décision du 8 octobre 2001 d'établir, au sein de l'Office of the President, l'Office of Homeland Security (OHS), chargé de développer et coordonner une stratégie globale de défense du territoire contre des menaces ou des attaques terroristes, s'inscrit totalement dans cette logique de gestion bureaucratique des problèmes. Elle ne fait qu'ajouter, à l'architecture déjà complexe de l'anti-terrorisme américain, un organe supplémentaire sans résoudre les problèmes liés au statut de Lead Agency. Son directeur, le Gouverneur Tom Ridge, n'est en effet doté, pour l'heure, d'aucune autorité opérationnelle. A cela s'ajoute l'implication, via l'Homeland Security Council, d'un nouvel acteur dans les affaires dites de sécurité intérieure: le département de la Défense. C'est ici une évolution majeure qui, d'une part, offre au département de la défense une place tout à fait prépondérante et, d'autre part, illustre l'interpénétration entre sécurité intérieure et extérieure.

L'implication grandissante du FBI dans l'anti-terrorisme est symptomatique de la criminalisation du terrorisme depuis 1984. En récupérant certaines prérogatives en matière d'anti-terrorisme, le FBI diffuse, en l'appliquant à ses domaines de compétence, sa propre définition du terrorisme qui n'effectue aucune distinction entre l'auteur d'une violence à motivation politique et un criminel.

Le terrorisme est devenu une sorte de prétexte pour alimenter les budgets. Les créations de services s'accompagnent en effet systématiquement d'une allocation de budgets. Le General Accounting Office (GAO) a ainsi dénoncé à plusieurs reprises l'utilisation anarchique et opaque des fonds alloués pour la lutte anti-terroriste.

La prédominance des acteurs de la sécurité dans le traitement, en amont, de la question terroriste. Chargés de la sécurité des individus ou du territoire, ils sont tentés d'agir sur 
les expressions d'un problème donné, mais jamais sur la racine du mal : des contextes sociaux pathologiques favorables à l'émergence d'une violence politique.

L'intérêt coupable porté aux nouvelles technologies s'est fait au détriment des méthodes classiques de renseignement. Trop occupés à développer le réseau Echelon et à analyser la "cyber-menace", les services de renseignement américains ont été incapables de prévenir le détournement de quatre avions par des pirates de l'air armés de cutters. C'est n'est pas ici la capacité des services de renseignement à collecter des informations qui est remise en cause mais, d'une part, l'utilité d'une collecte à si grande échelle et, d'autre part, leur capacité à analyser les données recueillies. Les attentats du 11 septembre n'ont en rien modifié cette tendance comme le prouve, d'abord, la création d'un poste de conseiller du Président pour les questions de cyber-sécurité et, ensuite, le vote de la loi anti-terroriste au mois d'octobre dernier qui élargit les compétences du FBI et de la NSA en matière d'écoutes téléphoniques et d'interception des messages électroniques.

Ces vingt dernières années, le terroriste est devenu l'une des nouvelles figures du discours sur la menace qui pèse sur les Etats-Unis et leurs alliés. Avec celles de l'immigré ou du trafiquant de drogue, elle a progressivement remplacé celle du défunt ennemi soviétique. L'attention s'est ainsi déplacée vers des individus regroupés en organisations dites terroristes - justifiant, au passage, l'implication du FBI - tandis que l'armée, via la doctrine Lake, s'est attachée, pour asseoir son rôle, à donner une forme étatique à cet ennemi (le Rogue State).

\section{NOTES}

1. . Cet article présente et actualise les axes principaux de la première partie d'un mémoire de DEA auquel on pourra se référer pour toutes les sources relatives aux définitions et autres chiffres cités dans ces pages : Philippe Bonditti, L'Anti-terrorisme aux Etats-Unis. De la menace soviétique aux Rogue States, la construction de l'ennemi terroriste aux Etats-Unis depuis 1980, mémoire de DEA, sous la direction de Didier Bigo, IEP de Paris, septembre 2001.

2. . Pour une approche en terme de champ de la sécurité, voir Didier Bigo, «L'Europe de la sécurité intérieure. Penser autrement la sécurité », in Anne-Marie Le Gloannec (dir.), Entre Union et Nations. L'Etat en Europe, Paris, Presses de Sciences Po, 1998, pp.85-87.

3. . Patterns of Global Terrorism : 2000.

Disponible sur internet : http://www.state.gov/s/ct/rls/pgtrpt/2000/index.cfm? docid $=2419$

4. . Terrorism in the United States 1999, Terrorist Research and Analytical Center, National Security Division, Federal Bureau of Investigation, Washington, DC, U.S. Department of Justice, 1999, p. ii.

5. . United States Departments of the Army and the Air Force, Military Operations in Low Intensity Conflict, Field Manual 100-20/Air Force Pamphlet 3-20, Washington, DC : Headquarters, Department of the Army and the Air Force, 1990, chap. 3, p.1.

6. . Voir à ce propos : http://www.cia.gov/terrorism/ctc.html 
7. . Patterns of International Terrorism : 1980.

8. . Voir à ce propos le rapport du General Accounting Office (GAO) intitulé Combating Terrorism, Comments on Counterterrorism Leadership and National Strategy, GAO, GAO-01-556T, March 27, 2001. Il recommande qu'une seule entité nationale dirige les programmes de lutte contre le terrorisme et développe la stratégie nationale à adopter. 9. . Pour tous ces chiffres, voir les rapports du GAO intitulés Combating Terrorism et notamment le rapport Combating Terrorism : FBI's Use of Federal Funds for Counterterrorism-Related Activities, GAO-GGD-99-7, novembre 1998.

10. . Lire à ce propos, Mickaël T. Klare, Low Intensity Warfare, New York, Pantheon Books, 1988.

11. . Conflits de basse intensité ou Low Intensity Conflicts (LIC).

12. . Voir notamment Mickaël T. Klare, Rogue States and Nuclear Outlaws, New York, Hillang Wang, 1995.

\section{RÉSUMÉS}

Analysant l'histoire et les dynamiques de l'antiterrorisme américain, Philippe Bonditti met l'accent sur les logiques bureaucratiques et politiques des organismes de lutte anti-terroriste pour expliquer les dynamiques de construction et d'aperception des menaces. Dans cet article, l'auteur montre par ailleurs comment la structuration des services de lutte antiterroriste américains et les procédures de coordination ont renforcé les compétitions entre les organismes pour dire et combattre la menace terroriste.

Philippe Bonditti analyses the history and the dynamics of the American antiterrorism and highlights the bureaucratic and political logic of organisations fighting against terrorism to explain the dynamics of threat construction. The author shows how the structuration of the American antiterrorist services and the co-ordination procedures have reinforced competition between organisations to declare and fight terrorism.

\section{INDEX}

Mots-clés : bureaucraties/ théorie des organisations, lutte anti-terroriste, politiques publiques, Relations Internationales

Thèmes : CIA, Federal Bureau of Investigation (FBI), Pentagone (US Department of Defence)

Index géographique : Etats-Unis 